\title{
La evaluación de las competencias en Educación Patrimonial del alumnado del Grado de Historia de la Universidad de Málaga'
}

Enviado: 3 de abril de 2020 / Aceptado: 20 de mayo de 2020

Publicado: 30 de diciembre de 2020

\author{
ANTONIO RAFAEL FERNÁNDEZ PARADAS \\ Departamento de Didáctica de las Ciencias Sociales \\ Universidad de Málaga, España \\ antonioparadas@ugr.es \\ MERCEDES FERNÁNDEZ PARADAS \\ Departamento de Historia Moderna y Contemporánea \\ Universidad de Málaga, España \\ paradas@uma.es
}

ISRAEL DAVID MEDINA RUIZ

Doctorado Interuniversitario en Patrimonio de las Universidades de Jaén, Córdoba, Extremadura y Huelva

Universidad de Jaén, España

idmr0001@red.ujaen.es

DOI 10.24310/IJNE3.2.2020.8451

\section{RESUMEN}

Este artículo analiza el nivel de competencias en Educación Patrimonial del alumnado del Grado de Historia de la Universidad de Málaga, concretamente al alumnado matriculado en el tercer curso del grado de Historia, con un total de 186 alumnos participantes en esta encuesta. Este análisis resulta necesario como paso previo para poder implementar estrategias dirigidas a la mejora de la enseñanza-aprendizaje del Patrimonio. De esta manera, hemos podido identificar las fortalezas y debilidades de los estudiantes de

\section{ABSTRACT}

The evaluation of the competences in Heritage Education of students of the Degree of History of the University of Malaga

This paper aims at analysing students' level of competence in Heritage Education within the Degree in History at the University of Malaga, specifically students enrolled in the third year of the History Degree, with a total of 186 students participating in this survey, as a preliminary step required to implement strategies

1 Este texto forma parte de los resultados de los proyectos de innovación educativa, "Evaluación de Competencias a través de TIC" de la Universidad de Jaén, e "Implementación de mejoras en el proceso de enseñanza-aprendizaje de la Educación Patrimonial en Humanidades, Ciencias Sociales y Educación", Código 19-036 de la Universidad de Málaga. 
esa titulación en Educación Patrimonial. Para el desarrollo de la investigación, siguiendo una metodología científica mixta cuantitativa y cualitativa de carácter interpretativo, hemos diseñado un cuestionario que han cumplimentado los alumnos y alumnas del Grado, matriculados en la asignatura obligatoria Historia Contemporánea de España durante el curso académico 2019-2020. El cuestionario persigue principalmente tres objetivos: 1) Hacer un perfil del estudiantado; 2) Valorar el grado de formación que ha recibido sobre Patrimonio antes de los estudios universitarios y en el Grado de Historia; y, 3) Su nivel de conocimiento y uso acerca de recursos en Internet y de las Tecnología de la Información y de la Comunicación en relación al Patrimonio. Hemos analizado las respuestas, de las que se colige la escasa presencia de la Educación Patrimonial en dicha titulación, por tanto, la necesidad de realizar acciones en ese sentido, tales como curso 0 sobre Patrimonio al inicio del primer curso académico, organización de jornadas sobre esta materia e incorporarlo en las guías docentes tanto en el temario como en las actividades programadas.

Palabras Clave: competencias, evaluación, patrimonio, tecnologías de la información y la comunicación, universidad, título universitario de historia, cuestionario. aimed at improving the teaching-learning of Heritage. Thus, students' strengths and weaknesses are identified in relation to Heritage Education in their degree. To carry out this research, following a quantitative and qualitative mixed scientific methodology, a questionnaire is designed to be completed by the undergraduate students who enrolled in the compulsory course "Contemporary Spanish History" during the academic year 2019-2020. The questionnaire pursues three objectives: 1) Obtaining a general student profile; 2) assessing their level of education in relation to Heritage before entering university and the Degree in History; and 3) their level of knowledge and use of Internet resources and ICTs in relation to Heritage. The results show a scarce presence of Heritage Education in the aforementioned degree, hence the need to carry out actions in this regard, such as course "0 on Heritage" at the beginning of the first academic year, holding conferences on this topic and incorporating Heritage in teaching guides, both in the syllabi as well as in programmed activities.

Keywords: competence, assessment, heritage, information and communication technologies (icts), university, academic degree in history, questionnaire.

\section{INTRODUCCIÓN}

La Educación Patrimonial consiste en "Educación desde y hacia el patrimonio" (Fontal, 2016, p. 432). La Educación Patrimonial resulta fundamental para lograr una formación holística que posibilite el desarrollo de una ciudadanía consciente de su herencia patrimonial, material e inmaterial, así como promover sociedades democráticas y tolerantes (Arjones, 2017).

El concepto de Patrimonio es el resultado de un largo proceso que comenzó en el siglo XVIII, en el que tuvieron lugar tres novedades: $1^{\text {a }}$. La creación por los anticuarios de repertorios históricos ilustrados en los que valoraron los elementos patrimoniales medievales; $2^{\mathrm{a}}$. El hallazgo de Pompeya y Herculano, acontecimientos que permitieron la formación de colec- 
ciones de antigüedades que fueron el origen de futuros museos públicos; y $3^{\mathrm{a}}$. Excavaciones $\mathrm{y}$ otros descubrimientos arqueológicos que motivaron a los gobiernos a protegerlo, como forma de ganar prestigio, también por su valor económico. Fue el comienzo de un proceso de estudio, identificación y catalogación de periodos y estilos que contribuyeron a crear la idea de que los restos materiales de la historia constituyen la identidad de un pueblo. A finales de esa centuria, la Revolución Francesa trajo consigo el inicio de un nuevo concepto del Patrimonio que dejaría de ser inherente a la propiedad privada y a las clases privilegiadas para ser considerado un bien que debía ser disfrutado por todos. En el novecientos avanzó el reconocimiento de la singularidad de cada cultura y, con ello, de la diversidad cultural (Fontal, 2003).

En las últimas décadas se ha progresado en un enfoque lo más integrador posible sobre el Patrimonio, recogido en el Plan Nacional de Educación y Patrimonio del Gobierno de España de 2013. En ese documento se revisaron los anteriores Planes Nacionales de Patrimonio Cultural, introduciéndose el binomio patrimonio y educación, por tanto, la necesidad de incorporar contenidos patrimonialistas en el currículo de todos los niveles del sistema educativo (Fontana e Ibáñez, 2015). Así también en la educación no formal, donde instituciones patrimoniales españolas como museos, bibliotecas y archivos, desarrollan actividades didácticas con el patrimonio que conservan (Cuenca et al., 2014). En concreto, en el ámbito de la educación formal se persigue la implementación de la Educación Patrimonial en la normativa educativa, la mayor presencia de los contenidos patrimoniales en los currículos, la mejora de la capacitación de los docentes y de los recursos didácticos dirigidos a la transmisión del Patrimonio Cultural (Ministerio de Educación, Cultura y Deporte, 2016).

Compartimos la definición de Patrimonio de Martín Cáceres, percibido como un "hecho sociocultural constituido de manera holística, por diversas manifestaciones de carácter histórico, artístico, científico-tecnológico, etnológico y medioambiental” (2012, p. 105). De esta diversidad se deriva la necesidad de diferenciar entre Patrimonio Arqueológico, Patrimonio Artístico, Patrimonio Documental, Patrimonio Industrial, Patrimonio Inmaterial, Patrimonio Medioambiental... También conlleva que el Patrimonio es un espacio multidisciplinar que puede ser analizado desde campos de investigación diferentes, como historia, historia del arte, geografía, geología, biología, etc. (Cambil y Fernández, 2017, p. 33). Siendo así que podríamos establecer la educación patrimonial como una "disciplina nuclear en la gestión del patrimonio” (Fontal e Ibáñez, 2015, p. 18).

En esta investigación partimos de la premisa de que si pretendemos promover la Educación Patrimonial del alumnado del Grado de Historia previamente es imprescindible conocer sus conocimientos y competencias en esta materia. Con esa información será posible diseñar 
estrategias dirigidas a mejorar su proceso de enseñanza-aprendizaje. Somos conscientes de que como profesores debemos focalizar más nuestro trabajo docente hacia el Patrimonio, desafortunadamente no disponemos de datos concretos de cuáles son las carencias del estudiante en relación a la Educación Patrimonial, no se han desarrollado estudios en ese sentido, tampoco para los discentes de los Grados de Historia. De ahí, la necesidad de plantear esta investigación.

\section{MATERIAL Y MÉTODO}

Para este estudio nos hemos decantado por la combinación de técnicas de investigación cuantitativa, motivado por el interés de una rigurosidad en el proceso de investigación y en la recogida de información de manera estructurada y sistemática (Del Canto y Silva, 2013, p. 28), y cualitativa. La utilización de la encuesta es una de las técnicas más utilizadas para la obtención de información, siendo este el principal motivo para el uso de esta metodología mixta en este estudio. Para ello, se diseñó un cuestionario online semiestructurado, combinando preguntas abiertas y cerradas (Abascal y Grande, 2005, p. 26), aunque con predominio de preguntas cerradas, las cuales tienen como ventaja la fácil respuesta y codificación (Casas, Repullo y Donado, 2002, p. 150). Dicho cuestionario fue cumplimentado por los alumnos matriculados en la asignatura de Historia Contemporánea de España, de carácter obligatoria y de tercer curso, impartida en el Grado Historia de la Universidad de Málaga durante el curso académico 2019/2020, pudieron acceder a la misma desde el Campus Virtual de la asignatura un total de 186 alumnos.

El cuestionario incluye tres tipos de preguntas: una pregunta de texto libre; preguntas para elegir entre más de dos opciones y preguntas con respuesta de sí o no.

Las preguntas se agrupan en cuatro bloques. El primero, dedicado a las características del estudiante, con el propósito de identificar si en general hay un único perfil o no. El segundo, acerca del nivel de formación que el alumno ha recibido sobre Patrimonio antes de los estudios universitarios y en el Grado de Historia. El tercero, planteado para aproximarnos a su grado de conocimiento de las distintas manifestaciones del Patrimonio (Patrimonio Documental, Industrial, Medioambiental...). El cuarto, dirigido a evaluar su grado de conocimiento y uso de recursos en Internet y de las Tecnología de la Información y de la Comunicación vinculados al Patrimonio.

El cuestionario consta de 32 ítems, en los que hemos pretendido reunir información sobre los siguientes aspectos:

$4 \cdot$ International Journal of New Education | Nº 
1. Perfil-es de los encuestados.

2. Definición del concepto de Patrimonio.

3. Formación recibida sobre Patrimonio antes de los estudios universitarios.

4. Formación recibida sobre Patrimonio en el Grado de Historia sobre Patrimonio y Educación Patrimonial, diferenciado por tipologías de Patrimonio.

5. Formación recibida sobre Patrimonio en el Grado de Historia en relación con determinados colectivos.

6. Conocimiento de la legislación sobre Patrimonio.

7. Si en el Grado hay una asignatura específica sobre Patrimonio.

8. Realización de trabajos de investigación histórica sobre Patrimonio.

9. Conocimiento y nivel de uso de recursos en Internet sobre Patrimonio.

10. Conocimiento de asociaciones vinculadas con el Patrimonio.

11. Evaluación por el profesorado de actividades en las que es preciso utilizar las competencias en Educación Patrimonial.

12. Empleo por el profesorado de las Tecnologías de la Información y la Comunicación para la enseñanza del Patrimonio.

13. Disposición del alumnado para mejorar su formación sobre Patrimonio.

14. Si conocimientos y competencias sobre Patrimonio pueden ser de utilidad para la vida laboral.

La encuesta, cumplimentada por discentes de tercer curso del Grado de Historia de la Universidad de Málaga, es representativa ya que han respondido el 84,6\% de los matriculados en ese curso y el 10,3\% en la titulación. La encuesta fue realizada durante los meses de febrero y marzo de 2020.

\section{RESULTADOS}

\subsection{Bloque 1. Características del estudiantado participante en la encuesta}

Como se ha mencionado anteriormente, en la encuesta han participado un total de 186 alumnos y alumnas. Un primer dato que nos ha llamado la atención es que la mayoría de los encuestados, el 68,4\%, son hombres y el 31,6\% mujeres (Figura 1). Este bajo porcentaje de muje- 
res frente a hombres pone en cuestión la idea tradicional de que las carreras de humanidades suelen ser cursadas mayoritariamente por féminas.

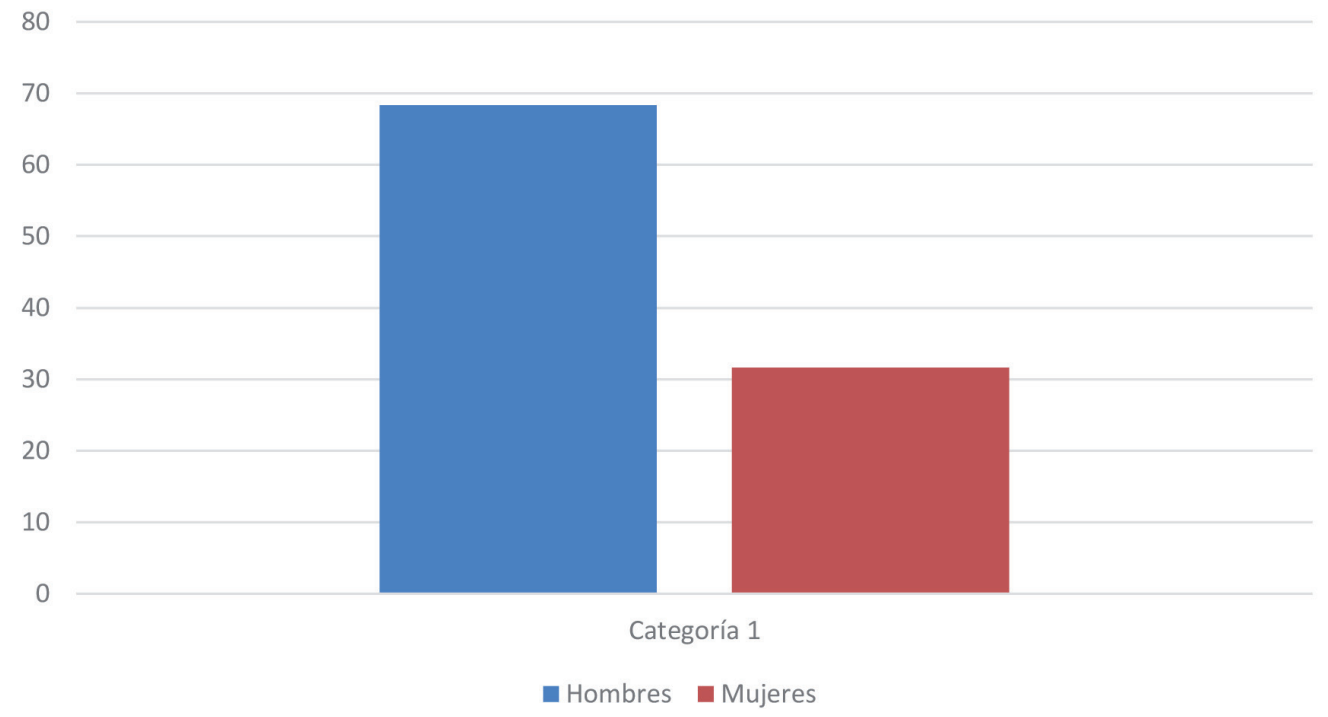

Figura 1. Alumnado participante en la encuesta, por sexos

En referencia al rango de edad de estos encuestados, el 33,3\% tienen 20 años, el 22,1\% 21 años y el 8,3\% 22 años, es decir, más de dos tercios están en la franja de edad que le corresponde para cursar el tercer año de la carrera. El 11,1\% de 23 a 25 años, el 16,7\% de 26 a 30 años y el 8,5\% tienen 31 años o más (Figura 2). Por tanto, en su inmensa mayoría son jóvenes los que han respondido al cuestionario. Cabe hacer mención en este punto que, como resultado de otra pregunta, hemos conocido que el $90 \%$ han accedido a los estudios universitarios desde el Bachillerato.

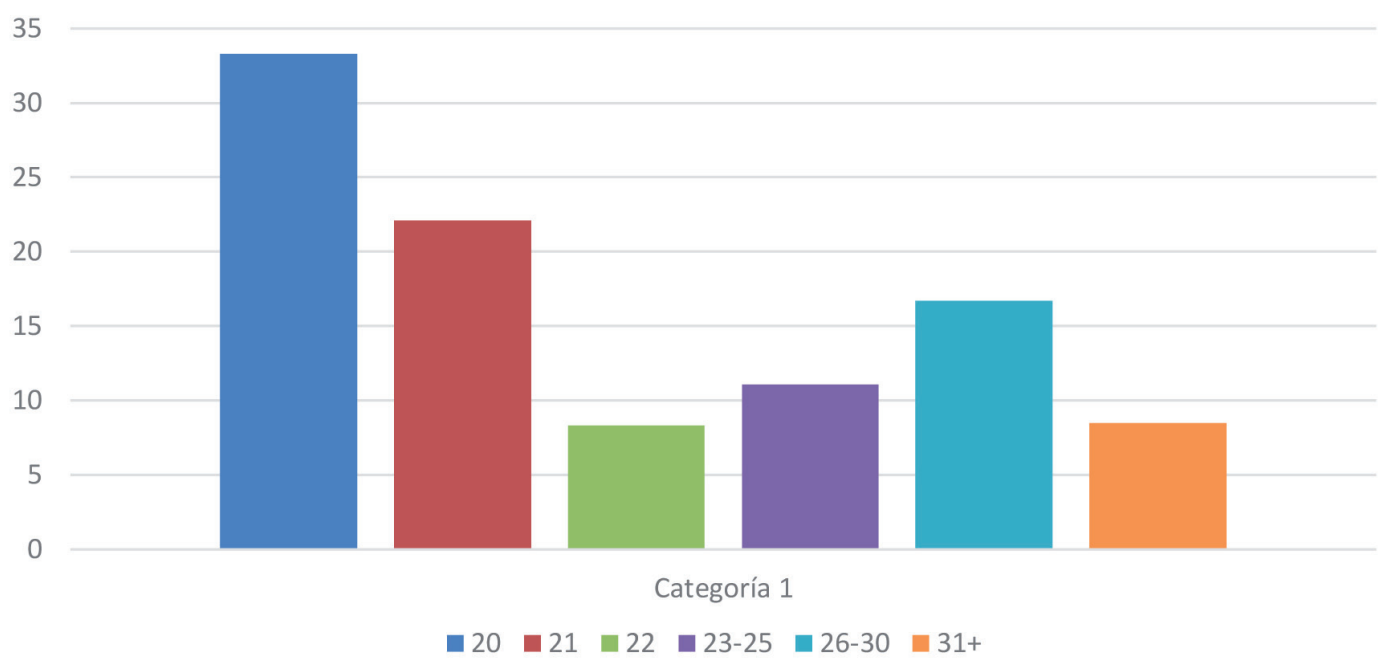

Figura 2. Rango de edad de los participantes 


\subsection{Bloque 2. Nivel de formación del alumnado en Patrimonio antes y durante el Grado en Historia}

A continuación, en la Tabla 1 se recoge cómo han definido el concepto Patrimonio los encuestados. De todas las respuestas obtenidas, hemos suprimido aquellas definiciones que eran similares desde un punto de vista conceptual y no aportaban información añadida relevante a las seleccionadas. Como se observa, se trata de un concepto subjetivo, donde la mayoría de los estudiantes no suelen definirlo correctamente, vinculándolos muchos de ellos con la propiedad. Sus respuestas nos indican que no son conscientes de las distintas manifestaciones del patrimonio.

Tabla 1. Definiciones de Patrimonio por los estudiantes del Grado de Historia de la Universidad de Málaga

\section{DEFINICIONES}

Conjunto de bienes de carácter material y cultural de interés que pertenecen a un Estado o población.

Aquellos restos materiales de culturas pasadas que son importantes muestras de la cultura de pueblos pasados o aquellas obras que han tenido una fuerte influencia cultural ya sea en su momento y también en el presente.

Propiedad no perteneciente a una persona que tiene fines didácticos y conmemorativos en pro de la investigación, docencia e información de un territorio, cultura o sociedad concretos.

Bienes propiedad de una persona o institución.

Patrimonio es todo aquello de valor económico, sea de una persona o de una institución.

Bienes muebles o inmuebles, culturales o no, que una nación o cultura reclama como de importancia sobre su pasado, presente o futuro y legisla para su protección, estudio, investigación o mantenimiento cuando les conviene (si no es históricamente hablando, solo son bienes).

Conjunto de bienes de una persona o un colectivo.

Patrimonio es la herencia cultural de un pueblo, con lo que nos identificamos.

Todo aquello con valor histórico o cultural digno de ser protegido.

El patrimonio podría definirse como legado. Hay diferentes tipos de patrimonio, como, por ejemplo, histórico, cultural, etc.

Patrimonio, hasta donde tengo entendido etimológicamente proviene de pater y monium, (lo que se hereda del padre), aunque también tenemos el patrimonio cultural, herencia de la cultura de tiempos pasados, ya no solo material sino también folklore, por ejemplo, los verdiales.

Lugares, objetos o espacios no materiales que tiene alguna importancia y que pertenecen a todas las personas.

Fuente: Cuestionario sobre Patrimonio de los estudiantes del Grado en Historia de la Universidad de Málaga.

$$
\text { Elaboración propia. }
$$


Una de las cuestiones importantes de este bloque iba encaminada a saber cuáles eran los conocimientos previos del alumnado del Grado en Historia respecto a Patrimonio. Por ello, la respuesta a la pregunta planteada en la Figura 3 nos indica que un alto número de los encuestados, el 60,5\%, han recibido formación sobre Patrimonio en Bachillerato, mientras que el 2,6\% ha sido mediante un Curso 0 de la titulación o poco antes del inicio del Grado. Este alto porcentaje de formación en Bachillerato se debe a que, en primero de Bachillerato en Andalucía, se imparte la asignatura Patrimonio Cultural y Artístico de Andalucía I, evidenciándose sus resultados en esta encuesta.

Figura 3. Pregunta: ¿Antes de comenzar el Grado en Historia que estás cursando, has recibido algún tipo de formación sobre Patrimonio?

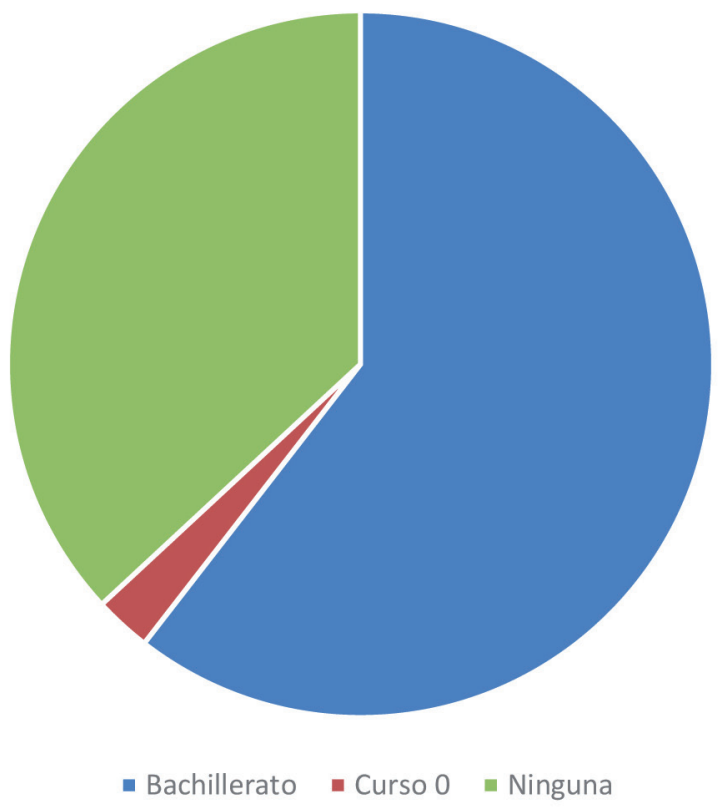

Respecto al mencionado Curso 0, estos alumnos deben referirse a cursos de formación no impartidos por la titulación, porque no se han organizado cursos 0 sobre Patrimonio para los nuevos estudiantes del título, o bien son discentes de otras universidades que están estudiando el curso en la Universidad de Málaga. El resto no ha recibido ningún tipo de formación.

Complementando estos datos formativos de los encuestados, el 52,6\% afirman que han recibido formación sobre Patrimonio en el Grado, cifra discreta para unos estudios tan vinculados al Patrimonio en sus diferentes acepciones. Este porcentaje va a disminuir al 12,2\% cuando responden a la cuestión relativa a si han recibido formación suficiente referente a Patrimonio.

Resulta curioso que cuando se les ha preguntado si han cursado en la titulación alguna asignatura específica sobre Patrimonio, el 10,5\% responde sí, lo cual contrasta con el hecho 
de que no existe una asignatura con ese perfil en el Grado en Historia de la Universidad de Málaga. Pensamos que este porcentaje puede haberse debido a dos motivos. El primero, la titulación incluye dos asignaturas obligatorias de historia del arte, Arte para Historiadores: el Arte Medieval y Renacentista de primer curso, y Arte para Historiadores: el Arte del Barroco al Mundo Actual, de segundo curso. Los estudiantes suelen confundir el Patrimonio con la historia del arte. El segundo, puede haber sucedido que algunos alumnos estén cursando la asignatura Historia Contemporánea de España merced a programas de intercambio y que en sus universidades de procedencia hayan cursado asignatura-s de Patrimonio.

Siguiendo en este aspecto formativo general sobre Patrimonio, también se les ha preguntado si han recibido preparación referente a las competencias en Educación Patrimonial. En la misma cuestión les hemos proporcionado la definición de este concepto, pues bien, solo el $21,1 \%$ opinan que han recibido preparación al respecto, tanto por ciento que desciende al $5,3 \%$ cuando les consultamos si la formación ha sido suficiente. A pesar de todas estas carencias visibilizadas en los datos, el 18,4\% responden positivamente cuando se les pregunta si han realizado algún trabajo de investigación sobre patrimonio en algún momento de sus estudios universitarios, no siendo este un porcentaje excesivamente bajo si consideramos la diversidad de temáticas por las que pueden haber optado.

Observadas y analizadas estas deficiencias formativas en Patrimonio por parte del alumnado del Grado en Historia, la cuestión ¿estarías dispuesto a realizar un taller sobre patrimonio?, adquiere una mayor relevancia. El $84,2 \%$ han respondido afirmativamente. Por tanto, pese a que la formación y el manejo de recursos por el estudiantado relacionados con el $\mathrm{Pa}$ trimonio deja mucho que desear, lo cual resulta especialmente preocupante en un Grado en Historia, sí parece que se muestra receptivo a mejorar sus carencias aunque no sea por la vía curricular propia del Grado en Historia, participando en acciones formativas complementarias para adquirir esos conocimientos que no han podido obtener por esta vía.

En una última cuestión planteada en el abordaje de este ámbito formativo en el alumnado, hemos intentado discernir si los estudiantes establecen una vinculación positiva entre mayor formación y competencias sobre Patrimonio y vida profesional actual y futura. El resultado de esta cuestión ha sido que casi el $90 \%$ consideran que puede ayudarle esta educación en Patrimonio en su desarrollo laboral.

\subsection{Bloque 3. Formación en distintas tipologías de Patrimonio}

Hemos querido ir más al detalle en cuanto a formación patrimonial en sus diferentes tipologías y no solo en educación patrimonial en general. Para ello, hemos realizado una serie de preguntas cuyas respuestas quedan plasmadas en la Figura 4. 
Figura 4. Formación de los encuestados en distintas tipologías de Patrimonio

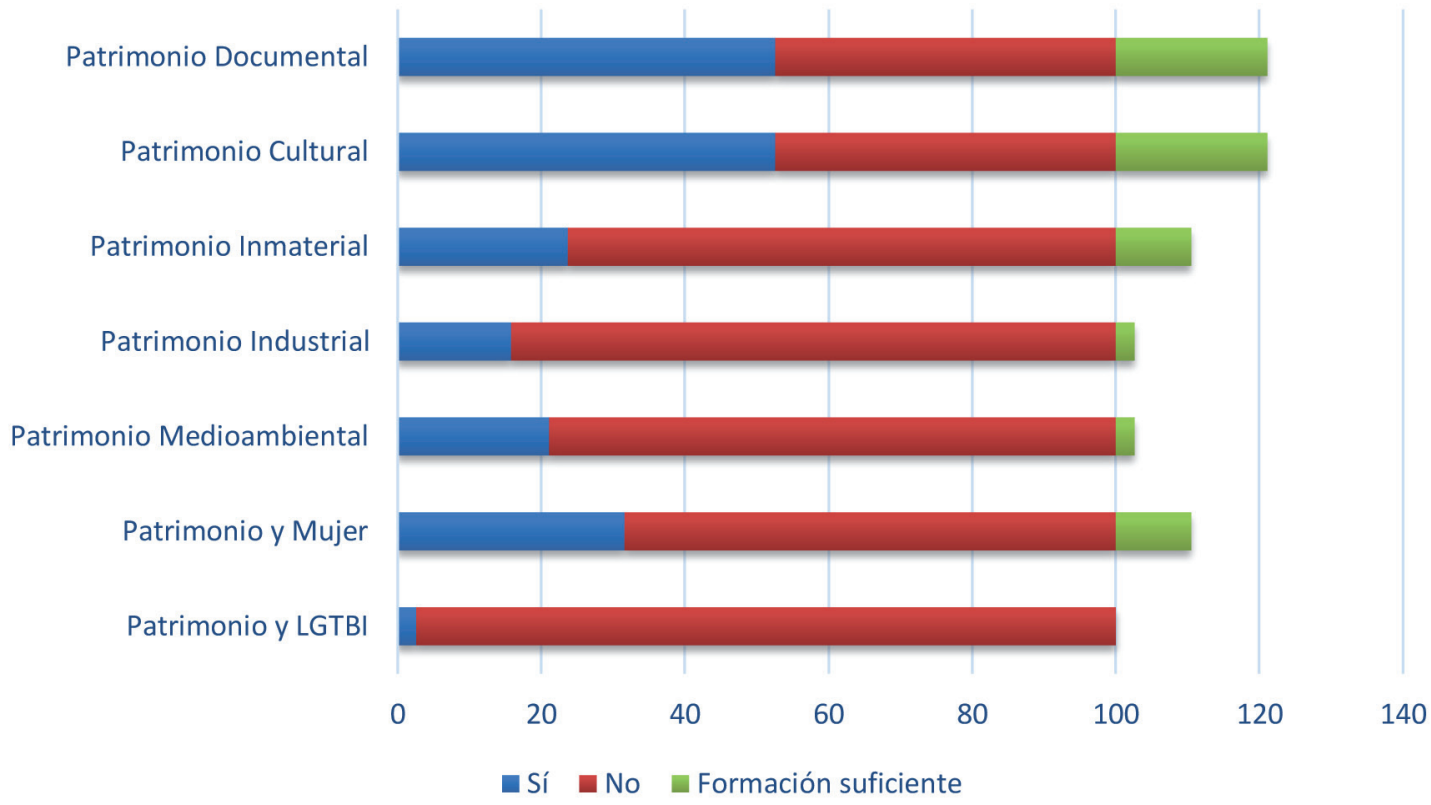

Primeramente, se ha indagado acerca de la formación recibida sobre Patrimonio Documental, el más próximo a la titulación que están cursando. La respuesta mayoritaria resulta desalentadora, el 52,6\% dicen que sí, guarismo que se reduce al 21,1\% al preguntarles sobre si la formación en Patrimonio Documental es suficiente. Los porcentajes de que los que dicen que han tenido algún tipo de formación sobre Patrimonio Cultural son similares, como podemos observar.

El panorama es todavía si cabe más desalentador cuando le interpelamos acerca de otras tipologías de Patrimonio. En lo concerniente al Patrimonio Inmaterial, solo el 23,7\% afirman que han recibido formación sobre el mismo, cifra que disminuye al 10,5\% cuando les preguntamos si esa formación ha sido suficiente Resultados más negativos hemos constatado cuando les interrogamos sobre el Patrimonio Industrial, el 15,8\% dicen que han recibido formación, solo el 2,6\% formación suficiente. En cuanto al Patrimonio Medioambiental, el 21,1\% responden positivamente, dato que merma al 2,6\% cuando se les pregunta si ha sido suficiente.

También hemos querido averiguar acerca de su formación sobre el Patrimonio vinculado a determinados colectivos. Respecto de las mujeres, la respuesta ha resultado más satisfactoria que las últimas reflejadas, el 31,6\% consideran que han recibido formación sobre patrimonio vinculada a ese grupo, porcentaje que baja al 10,5\% cuando les preguntamos si ha sido suficiente. Estos datos ponen de manifiesto la cada vez mayor inclusión de la mujer en el currículo de Historia. El dato más negativo de todas las consultas planteadas se refiere al colectivo LGT- 
BI (Lesbianas, Gais, Bisexuales y Transgénero) ya que solo el 2,6\% afirman haber recibido formación sobre Patrimonio relacionado con este grupo y el 100\% que no han recibido formación suficiente. Esto es algo que contrasta con el creciente desarrollo de la legislación autonómica destinada a promover y conservar el Patrimonio de ese colectivo (Fernández, 2020). Por tanto, se trata de uno de los asuntos que habría que reforzar con mayor intensidad al promover estrategias de aprendizaje del Patrimonio.

\subsection{Bloque 4. Nuevas Tecnologías y Patrimonio en el proceso enseñanza-aprendizaje}

El siguiente grupo de preguntas se refiere al nivel de conocimiento y uso de recursos por el estudiantado de Internet y de las Tecnología de la Información y de la Comunicación en relación al Patrimonio. Un recurso del que, en los últimos años, autores como Subires (2012) o Cambil y Romero (2016), han realizado sendos estudios referidos a la importancia de Internet como preservador del patrimonio cultural inmaterial. Para tal fin vamos a dividir los datos en dos vías: por parte del alumnado y por parte del profesorado. Esto es necesario porque si bien es importante conocer cómo el alumnado utiliza, si es que lo hace, estos recursos tecnológicos; más importante aún es conocer si el profesorado conoce y usa estos recursos para que sirvan de punto de partida en la educación patrimonial del alumnado, implementándolos luego ellos en su quehacer formativo y laboral futuro.

\section{a. Por parte del alumnado}

En general, en este apartado, las respuestas positivas son más elevadas en comparación con las anteriores referidas a formación en Patrimonio. Como apreciamos en la Figura 5, el 71,1\% dicen conocer Europeana, la biblioteca digital europea, de acceso libre, que reúne material digitalizado de los estados miembros de la Unión Europea. También se les ha preguntado sobre otras webs vinculadas al Patrimonio, aunque en este caso solo el 66,2\% dicen que conocen una web específica de esta temática. Esto es llamativo porque son alumnos de tercero, a solo poco más de un año de terminar su formación universitaria, donde lo razonable sería que estas cifras se acercaran al 100\%. Por su parte, al observar su conocimiento en webs concretas vemos que el 65,8\% conocen la web del Instituto Andaluz de Patrimonio Histórico. En relación a la web del Instituto de Patrimonio Cultural de España, poco más de un tercio afirman conocerla. 
Figura 5. Grado de conocimiento de webs especializadas en Patrimonio

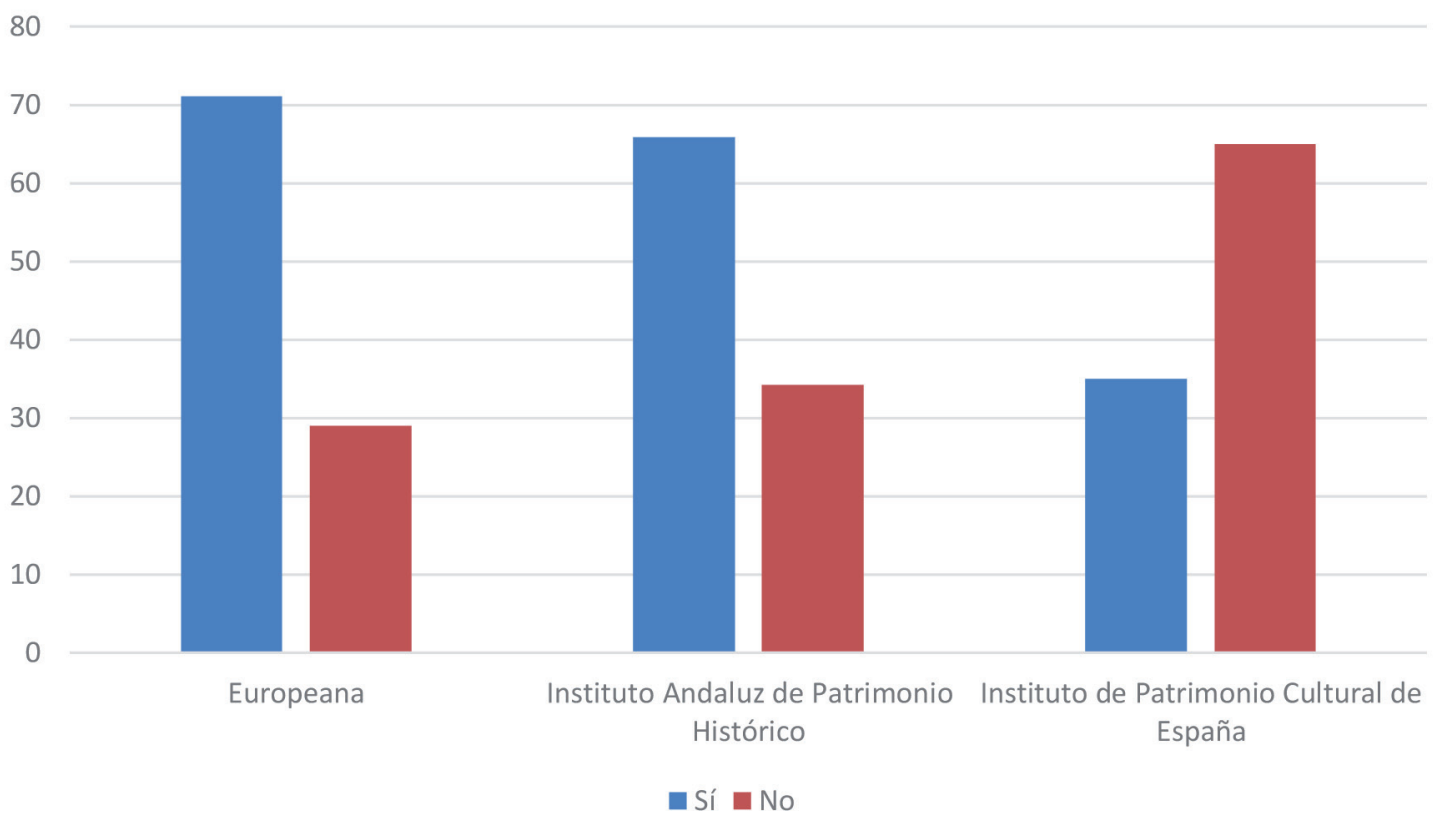

Figura 6. Grado de consulta de webs especializadas en Patrimonio en España

90

80

70

60

50

40

30

20

10

0

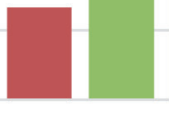

Europeana
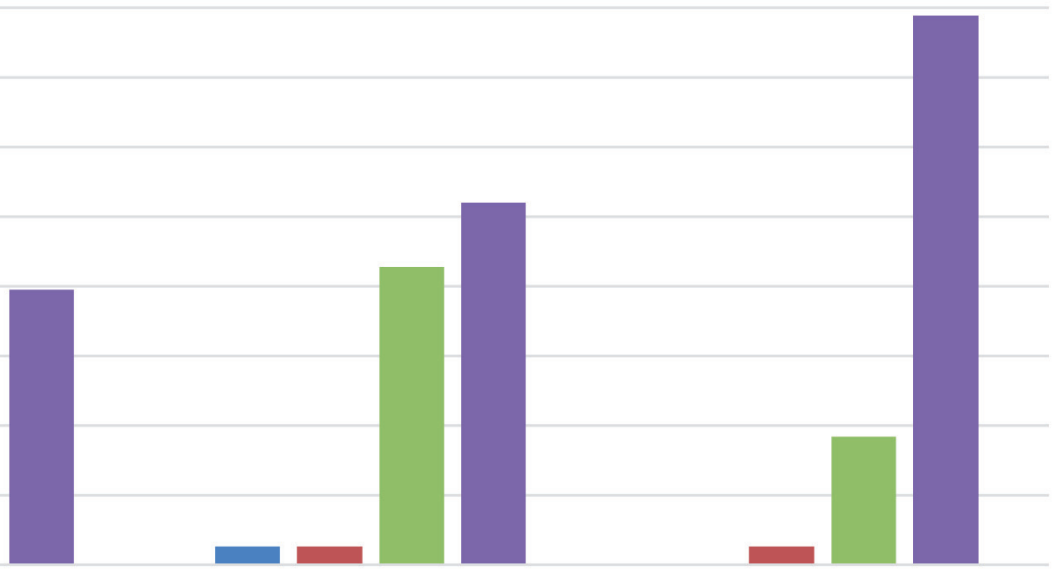

Instituto Andaluz de
Histórico

$$
\text { España }
$$

- Todas las semanas

- Una vez al mes

Pocas veces

Nunca

Ahora bien, si les consultamos acerca de la frecuencia de uso de estos recursos web, los datos decrecen. Como podemos observar en la Figura 6, en Europeana el 39,5\% afirman que nunca acceden, el 47,4\% que pocas veces, el 13,2\% una vez al mes y ninguno todas las semanas. Es decir, conocen este portal, pero apenas lo consultan. En el caso de la web del Instituto Andaluz de Patrimonio Histórico, el 52\% nunca la consultan, pocas veces el $42.8 \%$, una vez al mes el $2,6 \%$ y todas las semanas el mismo porcentaje. De nuevo, obser- 
vamos que el conocimiento de un recurso especializado no asegura que sea visitado por el alumnado. Para la web del Instituto de Patrimonio Cultural de España apreciamos unas cifras desalentadoras, debido a que el 78,9\% dicen que nunca la consultan, pocas veces el $18,4 \%$, una vez al mes el $2,6 \%$ y ninguno todas las semanas.

En lo concerniente a su conocimiento acerca de asociaciones vinculadas con el Patrimonio, el 55,3\% dicen que conocen una asociación dedicada a su defensa y el 44,7\% que conocen una asociación profesional dedicada a la gestión patrimonial.

Figura 7. Recursos didácticos usados en el aula

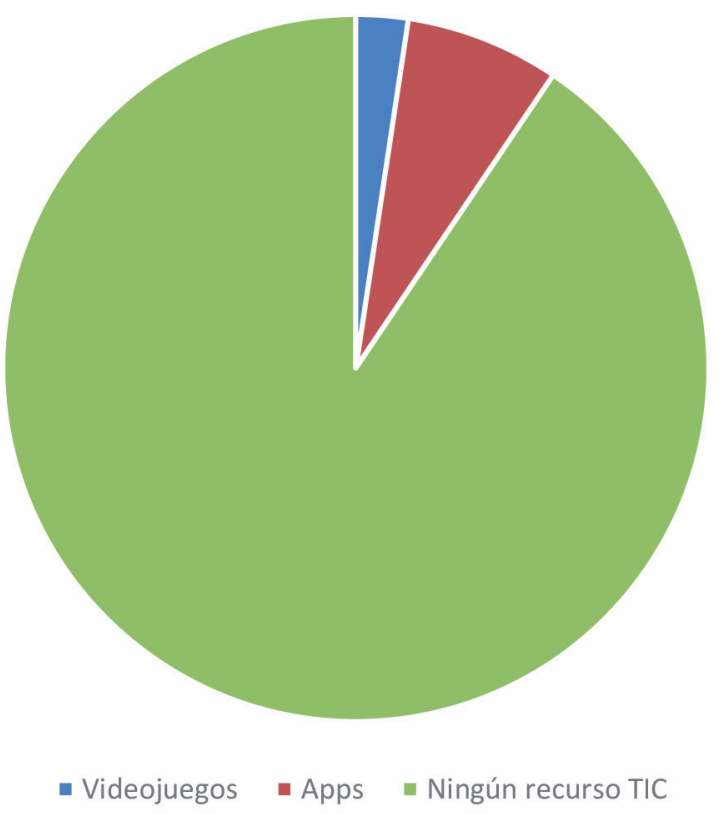

b. Por parte del profesorado

Resulta importante el hecho de la implementación en la práctica docente de recursos didácticos donde las tecnologías emergentes juegan un papel muy importante y de gran ayuda docente, especialmente en Patrimonio. Por ello, hemos querido conocer el grado de implicación que tienen estas tecnologías en el proceso de enseñanza-aprendizaje del alumnado encuestado por parte de sus docentes. Como podemos apreciar en la Figura 7, únicamente el $2.6 \%$ ha contestado de forma afirmativa al consultarles si sus docentes han recurrido al videojuego para enseñar Patrimonio. El uso del videojuego como recurso educativo es algo cada vez es más utilizado en educación secundaria y bachillerato, aunque se evidencia que no es un recurso utilizado en la enseñanza superior, al menos en los estudios del Grado que nos ocupa. 
De igual forma, les hemos interrogado acerca de si los profesores utilizan las apps en su práctica docente para la enseñanza del Patrimonio, sólo el 7,7\% han contestado positivamente.

Con todo, las respuestas a estas cuestiones muestran, de manera indirecta, el escaso empleo de las Tecnologías de la Información y la Comunicación por los docentes del Grado de Historia de la Universidad de Málaga.

Si nos detenemos en cómo se realiza el proceso de la evaluación de la competencia en Patrimonio, se les hizo una pregunta referida a si en el caso de que el profesorado evaluara una actividad en la que ha sido necesario recurrir a las competencias en educación patrimonial se han incluido rúbricas para tal fin. El 78,9\% de los encuestados responden desconocerlo, el $10,5 \%$ lo hacen positivamente y el 10,6\% negativamente. Por lo tanto, esto indicaría que, en general, los docentes no están considerando la evaluación en competencias patrimoniales.

\section{CONCLUSIONES}

Del análisis de los datos recabados de las respuestas del cuestionario, se deduce que el nivel de conocimientos y de competencias del alumnado del Grado en Historia de la Universidad de Málaga es muy insuficiente en Patrimonio, prácticamente en todos los temas consultados, lo cual resulta especialmente grave al considerar que debe ser un aspecto fundamental de su formación como graduado en Historia. También se dejará notar cuando se incorporaren al mercado laboral, lo que puede limitar su desarrollo profesional.

La formación recibida antes y durante la titulación es manifiestamente mejorable. Es relativamente positivo que más del $60 \%$ afirmen que han recibido formación sobre patrimonio en Bachillerato pero cuando se les pregunta si ha sido suficiente, el porcentaje se reduce drásticamente al 2,6\%. Respecto de la recibida en la titulación, poco más de la mitad responden que sí, cifra que desciende al 21,1\% al preguntarles si es suficiente En relación a la formación en competencias en Educación Patrimonial, los datos son aún más negativos.

$\mathrm{Al}$ interrogarles sobre las distintas tipologías de Patrimonio, en ningún caso más del $22 \%$ afirman que han recibido formación al respecto, porcentaje que desciende considerablemente al preguntarles acerca de si la formación ha sido suficiente, incluso en lo concerniente al Patrimonio Documental.

En cuanto al nivel de conocimiento y uso por parte de los estudiantes de Internet y de las Tecnología de la Información y la Comunicación en relación al Patrimonio, en general las 
respuestas dadas muestran mejores resultados. Por ejemplo, más de dos tercios conocen Europeana y las webs de Instituto del Patrimonio Histórico Andaluz y del Patrimonio Cultural de España. Ahora bien, su grado de uso de las mismas es poco frecuente.

En lo concerniente a su conocimiento sobre asociaciones vinculadas al Patrimonio, en torno a la mitad dicen conocer alguna, lo que también refleja su escaso desarrollo de la conciencia patrimonial, también de que puede ser un espacio en el que desarrollarse profesionalmente.

En general, el profesorado no está evaluando las competencias en Educación Patrimonial mediante rúbricas, lo que de manera indirecta pone de manifiesto que no está diseñando actividades sobre Patrimonio para el alumnado. Tampoco suele recurrir a las tecnologías emergentes, como videojuegos y apps, que pueden resultar especialmente útiles y atractivas para los alumnos, focalizadas al aprendizaje del Patrimonio.

Por último, los estudiantes, más del $82 \%$, se muestran receptivos a realizar talleres específicos sobre Patrimonio y más del $90 \%$ estiman que esa formación puede serles de utilidad para su vida profesional. Desafortunadamente, desde el Grado de Historia de la universidad malacitana no se les está proporcionando el conocimiento ni fomentando las competencias necesarias en Educación Patrimonial. De esta conclusión, se infiere la necesidad de organizar un Curso 0 para el alumnado de primer curso al inicio del mismo, jornadas dirigidas a darles a conocer por expertos las distintas tipologías de Patrimonio, así como incorporar el Patrimonio de manera sistemática en el temario y las actividades.

\section{REFERENCIAS BIBLIOGRÁFICAS}

Abascal, E., Grande, I. (2005). Análisis de encuestas. Editorial Esic.

Arjones, A. (2017). La Educación y el Patrimonio Industrial de España: la protección del patrimonio industrial a través de la competencia en conciencia y expresiones culturales. En II Congreso Internacional Virtual sobre la Educación en el siglo XXI, 15-29 de marzo de 2019. Eumednet.

Calaf, R. (2009). Didáctica del patrimonio. Epistemología, metodología y estudio de casos. Editorial Trea.

Cambil Hernández, Mª . E. y Fernández Paradas, A. R. (2017). El concepto actual de Patrimonio Cultural y su valor educativo: fundamentación teórica y aplicación didáctica. En M. E. Cambil y A. Tudela (Eds.), Educación y patrimonio cultural. Fundamentos, contextos y estrategias didácticas (pp. 28-46). Pirámide. 
Cambil Hernández, Ma . E. y Romero, G. (2016). Las TIC, las TAC y Redes 3.0. para la enseñanza de las ciencias sociales. En A. Liceras y G. Romero (Eds.), Didáctica de las ciencias sociales. Fundamentos, contextos y propuestas (pp. 271-275). Pirámide.

Casas, J., Repullo, J.R., y Donado, J. (2003). La encuesta como técnica de investigación. Elaboración de cuestionarios y tratamiento estadístico de los datos. Atención Primaria, 31 (8), 527 - 538.

Cuenca López, J. M., Martín Cáceres, M. J., Ibáñez-Etxebarria, A. y Fontal Merilla, A. (2014). La educación patrimonial en las instituciones patrimoniales españolas. Situación actual y perspectivas de futuro. Clio. History and History teaching, 40.

Del Canto, E., y Silva, A. (2013). Metodología cuantitativa: abordaje desde la complementariedad en ciencias sociales. Revista Ciencias Sociales, 141 (3), 25-34. https://doi.org/10.15517/rcs. v0i141.12479

Estepa, J. (2009). La Educación del Patrimonio y la ciudadanía europea en el contexto español. En R. M. Ávila, B. Borghi y I. Mattozzi (Eds.), L'educazione alla cittadinanza europea e la formazione degli insegnanti (pp. 327-352). Pàtron.

Fernández Paradas, A. R. (2020). Patrimonio y memoria LGTBI en las leyes autonómicas. El profesional de la información, 29 (1). https://doi.org/10.3145/epi.2020.ene.10

Fontal, O. (2003). La educación patrimonial. Teoría y práctica en el aula, el museo e Internet. Editorial Trea.

Fontal (2016). Educación patrimonial: retrospectiva y prospectivas para la próxima década. Estudios Pedagógicos, 42 (2).

Fontal, O., e Ibáñez, A. (2015). Estrategias e instrumentos para la educación patrimonial en España. Educatio Siglo XXI, 33 (1), 15-32.

Fontal, O. y Marín, S. (2015). La educación patrimonial en España: necesidades y expectativas para la próxima década. Revista de Patrimonio, 85.

Hernández Ríos, Mª . L. (2017). La Educación Patrimonial en contextos de educación no formal: un desafío para maestros en formación. En M. E. Cambil y A. Tudela (Eds.), Educación y patrimonio cultural. Fundamentos, contextos y estrategias didácticas (pp. 217-238). Pirámide.

Martín Cáceres, M. J. (2012). La educación y la comunicación patrimonial: una mirada desde el Museo de Huelva (tesis doctoral, Universidad de Huelva). Repositorio Institucional UHU. http://hdl. handle.net/10272/6048

Ministerio de Educación, Cultura y Deporte (2016). Plan Nacional de Educación y Patrimonio del Gobierno de España. https://sede.educacion.gob.es/publiventa/plan-nacional-de-educacion-y-patrimonio/patrimonio-historico-artistico/20704C 
Morente, M. (2006). El concepto actual del patrimonio cultural. Boletín del Instituto del Patrimonio Histórico, 58, 40-43.

Subires Mancera, M. P. (2012). Cultura en la era Web 2.0. Cuadernos de comunicación e innovación, 91, 132-140. 